\title{
Psychological Implications of Unemployment Among Bangladesh Civil Service Job Seekers: A Pilot Study
}

\author{
Md. Abdur Rafi 1,2t, Mohammed A. Mamun ${ }^{2,3 \dagger}$, Kamrul Hsan ${ }^{3,4}$, Moazzem Hossain 4 \\ and David Gozal ${ }^{5 *}$
}

${ }^{1}$ Rajshahi Medical College, Rajshahi, Bangladesh, ${ }^{2}$ Undergraduate Research Organization, Dhaka, Bangladesh, ${ }^{3}$ Department of Public Health \& Informatics, Jahangirnagar University, Dhaka, Bangladesh, ${ }^{4}$ Institute of Allergy and Clinical Immunology of Bangladesh, Dhaka, Bangladesh, ${ }^{5}$ Department of Child Health and the Child Health Research Institute, The University of Missouri School of Medicine, Columbia, MO, United States

\section{OPEN ACCESS}

Edited by:

Sebastian von Peter,

Medizinische Hochschule Brandenburg Theodor Fontane,

Germany

Reviewed by:

Lingkan Barua,

Bangladesh University of Health

Sciences, Bangladesh

Md. Sahab Uddin,

Southeast University,

Bangladesh

${ }^{*}$ Correspondence:

David Gozal

gozald@health.missouri.edu

tThese authors have contributed equally to this work.

Specialty section:

This article was submitted to

Public Mental Health,

a section of the journal

Frontiers in Psychiatry

Received: 09 March 2019

Accepted: 22 July 2019

Published: 12 August 2019

Citation:

Rafi MA, Mamun MA, Hsan K, Hossain M and Gozal D (2019)

Psychological Implications of Unemployment Among

Bangladesh Civil Service Job Seekers: A Pilot Study.

Front. Psychiatry 10:578. doi: 10.3389/fpsyt.2019.00578
Background: Recent trends suggest that university graduates seeking jobs are more susceptible to common mental disorders, such as depression, anxiety, or stress. However, the mental health issues among unemployed graduates has not been explored in Bangladesh yet.

Aims: This study aimed to assess for the first time the prevalence and associated risk factors of depression, anxiety, and stress among Bangladesh Civil Service (BCS) job seekers. Three hundred four graduates residing in Rajshahi, Bangladesh, who were preparing to attend the $40^{\text {th }}$ BCS examination, the most sought-after employment opportunity in the country, were surveyed.

Methods: Measures included socio-demographics, field of study, and career-related variables, and the Bangla Depression Anxiety Stress Scale (DASS-21). Chi-square test, Fisher exact test, and binary logistic regression with "depression," "anxiety," and "stress" as the dependent variables were carried out to identify the factors associated with these.

Results: Overall, the prevalence of moderate to extremely severe depression, anxiety, and stress was $49.3 \%, 53.6 \%$, and $28.3 \%$, respectively, with no detectable differences between genders. Insecurity related to a BCS job $(\mathrm{OR}=0.41 ; \mathrm{Cl}=0.26-0.65, p<0.001$; ref: job insecurity), family and social pressure to obtain a BCS job (OR $=4.58 ; \mathrm{Cl}=1.67-12.56$, $p<0.001)$, and stress $(\mathrm{OR}=8.33 ; \mathrm{Cl}=4.47-15.51, p<0.001)$ emerged as independent predictors for depression. In addition, having part-time job was associated with anxiety $(\mathrm{OR}=2.38 ; \mathrm{Cl}=1.34-4.23, p=0.003)$, and security in a BCS job and serving the nation through this job were negatively associated with stress $(\mathrm{OR}=0.59 ; \mathrm{Cl}=0.35-0.98, p=$ 0.042 vs. $\mathrm{OR}=0.59 ; \mathrm{Cl}=0.36-1.00, p=0.05)$.

Conclusion: The relatively high rates of depression, anxiety, and stress among graduate job seekers should prompt implementation of market force initiatives that incorporate interventions related to the major risk factors uncovered herein.

Keywords: depression, anxiety, stress, job seekers, unemployed graduates, Bangladesh Civil Service

Abbreviations: BCS, Bangladesh Civil Service; UUE, Unemployment or underemployment; DASS, Depression Anxiety Stress Scale. 


\section{INTRODUCTION}

Achieving a university degree is nowadays considered an important key to professional and societal success. The number of university students has markedly increased over the last half century along with remarkable expansion of universities and their campuses, reflecting the high demand. The increased numbers of university graduates are then anticipated to enter the labor force, but since the job market is not expanding fast enough to cope with the increasing number of graduates, increased competition and job uncertainty have emerged (1). Consequently, the number of unemployed and underemployed university graduates has steadily increased, with additional new graduates entering the job market and the inability of governments and the private sector to provide appropriate employment solutions (2). The rising unemployment or underemployment is likely to impose adverse societal and personal consequences that are not necessarily appropriately addressed. Indeed, recent studies have shown that the situational factors driving either unemployment or underemployment (UUE) were quite diverse, and appeared to be associated with the risk of manifesting common mental health consequences, such as depression, anxiety, and stress (2-7). Moreover, these common mental health problems account for at least $90 \%$ of suicide cases. Indeed, such events have been linked to but not restricted to a large spectrum of psychological and psychiatric disorders encompassing conditions such as depression disorder, adjustment disorders, alcohol and drug dependency and addiction, anxiety disorders, schizophrenia and other psychoses, and sleep disorders to name a few (8-11). In the sense of unemployment, from recent review articles, it was suggested as an unmet factor for promoting the vulnerability of suicidal behaviors by adding to the impact of stressful life events and also of suicide by evaluating proximal suicide risk factors (i.e., mental illness, family conflict, dissatisfaction, etc.) (12-14).

UUE-related elements such as economic distress and joblessness, as well as job insecurity, frequently underlie feelings of failure, which in turn may lead to depression, stress, and anxiety (3-6). Moreover, the family and social pressures associated with job-seeking activities and higher expectations from university graduates also act as potential mediators of depression and stress disorders among university graduates exploring the job markets (2). In parallel, socio-demographic characteristics (i.e., female, unmarried, lower quality of life, family socioeconomic status, academic major, educational expense loan, and willingness to accept irregular employment) are also significantly associated with the risk for mental disturbances $(3,15)$.

In Bangladesh, the situation is remarkably comparable to current global trends. According to a report of the Bangladesh Bureau of Statistics (BBS), about $11.2 \%$ of youths with tertiary education were unemployed in the 2016-2017 economic year. This unemployment rate is nearly threefold higher than the national unemployment rate of $4.2 \%$ (16). At least 6.6 million Bangladeshi university graduates, who are a priori qualified for permanent jobs, are unable to secure an employment opportunity as the job market is not expanding fast enough to cope with the increasing number of graduates (1). Moreover, the socio-cultural structure in Bangladesh has evolved in such a way that university graduates will preferentially seek to become government employees rather than to become entrepreneurs. Accordingly, each year, an everincreasing number of graduates will try to obtain employment in the Bangladesh Civil Service (BCS), the most prestigious and sought-after governmental employer in Bangladesh, and pursue available cadre positions (note: a job holder in the BCS is referred to as a cadre). For example, a total of 346,440 candidates applied for 2,024 vacant positions during the $38^{\text {th }}$ BCS examination (i.e., 171 candidates per position) (17). Therefore, the vast majority of university graduates will falter and be unable to secure their aspirational job. Even in the context of multiple repeated yearafter-year attempts, this can have consequent adverse effects on productivity as well as potentially promote reduced self-esteem or more serious psychiatric disorders.

In these contextual settings, it has become apparent that the global prevalence of common mental disorders is rising, especially in middle- and low-income countries, and leads to considerable losses in health and productivity (18). A recent systematic review focused on Bangladesh showed that the prevalence of common mental health problems has been reported in a very wide range, from $6.5 \%$ to $31.0 \%$ among adults and from $13.4 \%$ to $22.9 \%$ among children (19).

We are unaware of any previous studies examining potential issues of mental health among university graduates seeking employment. Recent reports in the news of suicides associated with unemployment among educated individuals residing inside $(1,20)$ or outside $(12-14)$ of Bangladesh prompted us to carry out the present study which aimed to evaluate the prevalence of depression, anxiety, and stress among university graduate BCS job seekers, and potentially identify risk factors associated with such mental health issues.

\section{METHODS}

\section{Participants and Procedure}

The BCS examination is the most competitive job-related examination in Bangladesh, which aims to select civil servants and is run by the Bangladesh Public Service Commission. The recruited officers are called BCS cadres, where 27 types of cadres (including general, technical, and mixed) are defined in the context of the Civil Service of Bangladesh (21). The current crosssectional pilot study was conducted among university graduates living in Rajshahi, Bangladesh, who were preparing themselves to participate in the $40^{\text {th }} \mathrm{BCS}$ examination to become a cadre. The survey was conducted between August and October 2018. A total of 350 printed questionnaires were distributed among students at three randomly selected BCS preparation coaching centers. The questionnaire took about 30 minutes to complete and data from 315 participants were collected (response rate $90 \%$ ). After removal of incomplete questionnaires $(n=9), 304$ were retained for final analyses.

\section{Ethics}

This study was approved by the Institutional Review Board of the Institute of Allergy and Clinical Immunology of 
Bangladesh (IACIB), Savar, Dhaka, Bangladesh [ref. no: IRBIACIB/ CEC/07201801]. Verbal and formal written consent from all respondents were obtained before participating in the study. All subjects were also informed about the i) nature and purpose of the study, ii) procedure of the study, iii) right to refuse, and iv) right to withdraw from participating in the study. The participants did not gain any financial benefit from taking part in the study. Confidentiality of data and anonymity to the participants were ensured.

\section{Measures}

\section{Socio-Demographics}

Socio-demographics included age, sex, residence (city or village), relationship status (i.e., single, engaged in relationship, or married), and socioeconomic status. Socioeconomic status was appraised from monthly total family income, which was categorized into higher, middle, and lower income groups corresponding to annual income of $>$ BDT30000, BDT15000 to BDT30000, and < BDT15000, respectively.

\section{Study and Job-Related Questions}

Study and career-related questions included the type of higher learning institution (public university or colleges under national university), year of graduation, whether they were doing any part-time job ("yes" or "no" response), whether they attended the BCS examination before or not, cadre of choice, and factors behind choosing the BCS as a professional career. For accessing driving factors of BCS preference, multiple-choice responses were offered while covering probable options (e.g., security of the job, high salary, family and social expectation, to serve the nation). Regarding BCS cadre choice, multiple options were included (e.g., administrative, education, police).

\section{Bangla Depression Anxiety Stress Scale (DASS)}

This is a 21-item self-report inventory that provides scores on three subscales, i.e., depression (7 items), anxiety (7 items), and stress (7 items) (22). Each item was rated on a four-point Likert scale ranging from "never" (0) to "always" (3). In the present study, the Bangla version DASS-21 was used (23). Moderate, severe, and very severe were combined to denote a problematic score for depression, anxiety, and stress in this study. In the Bangla version, Cronbach's alpha for the depression, anxiety, and stress subscales were 0.99, 0.96 , and 0.96, respectively (23). For this study the Cronbach's alpha for the depression, anxiety, and stress subscales were $0.787,0.783$, and 0.834 , respectively, and the overall Cronbach's a was 0.919 .

\section{Statistical Analysis}

Statistical Package for Social Science (SPSS) version 22.0 was used for data analysis. Continuous variables, i.e., age, are not shown in the tables, but categorical variables are presented as descriptive statistics (e.g., frequencies, percentages, and Chisquare and Fisher exact test). All variables were entered into binary logistic regression with "depression," "anxiety," and "stress" as the dependent variables. The results of logistic regression were interpreted with $95 \%$ confidence intervals.

\section{RESULTS}

A total of 304 BCS candidates (mean age $24.2 \pm 1.6$ years; females: $170(55.9 \%)$ ) participated in the study. The majority of the respondents (78.3\%) were from rural areas and belonged to the middle socioeconomic group (59.9\%). The vast majority were single, with $14.1 \%$ being married and $16.4 \%$ involved in a relationship. About $57.6 \%$ of participants had graduated from colleges compared to $42.7 \%$ having graduated from a university. In addition, $77.3 \%$ were exclusively preparing for the BCS examination without taking on any part-time job, and for the majority $(82.2 \%)$, this was their first attempt to take the BCS examination (Table 1).

The overall prevalence of depression, anxiety, and stress among the participants was $49.3 \%, 53.6 \%$, and $28.3 \%$ respectively, with women more likely to report these symptoms compared to men, albeit without reaching statistical significance [prevalence of depression, anxiety, and stress was $\left(53.5 \%\right.$ vs. $44.0 \% ; X^{2}=2.70$, $\mathrm{df}=1, p=0.100),\left(55.9 \%\right.$ vs. $\left.50.7 \% ; X^{2}=0.79, \mathrm{df}=1, p=0.373\right)$, and (31.2\% vs. $\left.24.6 \% ; X^{2}=1.58, \mathrm{df}=1, p=0.208\right)$, respectively] (Table 1).

Participants doing any part-time job were more likely to suffer from anxiety $\left(69.6 \% ; X^{2}=9.13, \mathrm{df}=1, p=0.003\right)$. Moreover, the prevalence of depression was lower among the candidates who had chosen the BCS as a career for better job security $(37.9 \%$; $X^{2}=14.44$, df $\left.=1, p<0.001\right)$. In contrast, when the reason for choosing BCS was family and social pressure, the risk of experiencing depressive disorder was increased $\left(80 \% ; X^{2}=10.24\right.$, $\mathrm{df}=1, p<0.001)$. Moreover, those who had chosen the career to serve the nation were less likely to report stress $\left(22.6 \% ; X^{2}=\right.$ $3.83, \mathrm{df}=1, p=0.05)$. Depression and stress were significantly correlated $(p=0.000)$, but no association was found between depression and anxiety, and between anxiety and stress. (Table 1)

Predictors of depression, anxiety, and stress from the multivariate logistic regression analysis are shown in Table 2. Seeking BCS employment for family and social pressure reasons $(\mathrm{OR}=4.58 ; \mathrm{CI}=1.67-12.56, p<0.001)$ and suffering from stress $(\mathrm{OR}=8.33 ; \mathrm{CI}=4.47-15.51, p<0.001)$ emerged as independent risk factors of depression, whereas seeking BCS employment as reflecting a secure job was negatively associated with depression $(\mathrm{OR}=0.41 ; \mathrm{CI}=0.26-0.65, p<0.001)$. Regarding anxiety risk, engaging in a current part-time job $(\mathrm{OR}=2.38$; $\mathrm{CI}=1.34-4.23$, $p=0.003)$ emerged as an independent risk factor. Moreover, BCS for security of job and to serve the nation were negatively associated with stress $(\mathrm{OR}=0.59 ; \mathrm{CI}=0.35-0.98, p=0.042$ vs. $\mathrm{OR}=0.59 ; \mathrm{CI}=0.36-1.00, p=0.05)$ (Table 2).

\section{DISCUSSION}

Unemployment among university graduates is one of the major social problems in Bangladesh, and the unemployment rates have continued to increase, most likely due to insufficient job creation despite a consistently high economic growth over the last several years $(1,16)$. Considering that the mental health issues among unemployed graduates has not been explored in Bangladesh previously (24-32), the present study aimed at addressing the gap in potential links between depression, 
TABLE 1 | Distribution of variables among respondents by depression, anxiety, and stress.

\begin{tabular}{|c|c|c|c|c|c|c|c|c|c|c|c|c|c|}
\hline \multirow[t]{2}{*}{ Variables } & \multirow{2}{*}{$\begin{array}{c}\text { Total = } 304 \\
\text { n (\%) }\end{array}$} & \multicolumn{4}{|c|}{ Depression } & \multicolumn{4}{|c|}{ Anxiety } & \multicolumn{4}{|c|}{ Stress } \\
\hline & & Yes (\%) & $X^{2}$ value & df & $p$-value & Yes (\%) & $X^{2}$ value & df & $p$-value & Yes (\%) & $X^{2}$ value & df & $p$-value \\
\hline \multicolumn{14}{|l|}{ Socio-demographics } \\
\hline \multicolumn{14}{|l|}{ Gender } \\
\hline $\begin{array}{l}\text { Male } \\
\text { Female }\end{array}$ & $\begin{array}{l}134(44.1) \\
170(55.9)\end{array}$ & $\begin{array}{l}59(44.0) \\
91(53.5)\end{array}$ & 2.705 & 1 & 0.100 & $\begin{array}{l}68(50.7) \\
95(55.9)\end{array}$ & 0.795 & 1 & 0.373 & $\begin{array}{l}33(24.6) \\
53(31.2)\end{array}$ & 1.585 & 1 & 0.208 \\
\hline \multicolumn{14}{|l|}{ Permanent residence } \\
\hline $\begin{array}{l}\text { Village area } \\
\text { City area }\end{array}$ & $\begin{array}{c}238(78.3) \\
56(18.4)\end{array}$ & $\begin{array}{l}115(48.3) \\
29(51.8)\end{array}$ & 0.218 & 1 & 0.641 & $\begin{array}{l}132(55.5) \\
25(44.6)\end{array}$ & 2.133 & 1 & 0.144 & $\begin{array}{l}69(29.0) \\
15(26.8)\end{array}$ & 0.108 & 1 & 0.742 \\
\hline \multicolumn{14}{|l|}{ Relationship status } \\
\hline $\begin{array}{l}\text { Married } \\
\text { In a relationship } \\
\text { Single }\end{array}$ & $\begin{array}{l}43(14.1) \\
50(16.4) \\
210(69.1)\end{array}$ & $\begin{array}{c}20(46.5) \\
26(52.0) \\
103(49.0)\end{array}$ & 0.283 & 2 & 0.868 & $\begin{array}{c}22(51.2) \\
30(60.0) \\
110(52.4)\end{array}$ & 1.049 & 2 & 0.592 & $\begin{array}{l}13(30.2) \\
10(20.0) \\
63(30.0)\end{array}$ & 2.071 & 2 & 0.355 \\
\hline \multicolumn{14}{|l|}{ Socioeconomic status } \\
\hline $\begin{array}{l}\text { Lower class } \\
\text { Middle class } \\
\text { Upper class }\end{array}$ & $\begin{array}{c}51(16.8) \\
182(59.9) \\
71(23.4)\end{array}$ & $\begin{array}{l}29(56.9) \\
88(48.4) \\
33(46.5)\end{array}$ & 1.458 & 2 & 0.482 & $\begin{array}{l}27(52.9) \\
94(51.6) \\
42(59.2)\end{array}$ & 1.169 & 2 & 0.558 & $\begin{array}{l}19(37.3) \\
43(13.6) \\
24(33.8)\end{array}$ & 5.035 & 2 & 0.081 \\
\hline \multicolumn{14}{|l|}{ Study- and job-related questions } \\
\hline $\begin{array}{l}\text { Graduation institute } \\
\text { College } \\
\text { University }\end{array}$ & $\begin{array}{l}175(57.6) \\
129(42.4)\end{array}$ & $\begin{array}{l}85(48.6) \\
65(50.4)\end{array}$ & 0.098 & 1 & 0.754 & $\begin{array}{l}95(54.3) \\
68(52.7)\end{array}$ & 0.074 & 1 & 0.786 & $\begin{array}{l}52(29.7) \\
34(26.4)\end{array}$ & 0.413 & 1 & 0.521 \\
\hline \multicolumn{14}{|l|}{ Graduation year } \\
\hline $\begin{array}{l}2015 \text { to } 2012 \\
2016 \\
2017 \\
2018\end{array}$ & $\begin{array}{c}52(17.1) \\
52(17.1) \\
84(27.6) \\
116(38.2)\end{array}$ & $\begin{array}{l}30(57.7) \\
19(36.5) \\
44(52.4) \\
57(49.1)\end{array}$ & 5.173 & 3 & 0.160 & $\begin{array}{l}29(55.8) \\
23(44.2) \\
45(53.6) \\
66(56.9)\end{array}$ & 2.441 & 3 & 0.486 & $\begin{array}{l}20(38.5) \\
14(26.9) \\
25(29.8) \\
27(23.3)\end{array}$ & 4.227 & 3 & 0.238 \\
\hline \multicolumn{14}{|l|}{ Current part-time job } \\
\hline $\begin{array}{l}\text { Yes } \\
\text { No }\end{array}$ & $\begin{array}{c}69(22.7) \\
235(77.3)\end{array}$ & $\begin{array}{c}31(44.9) \\
119(50.6)\end{array}$ & 0.696 & 1 & 0.404 & $\begin{array}{c}48(69.6) \\
115(48.9)\end{array}$ & 9.127 & 1 & 0.003 & $\begin{array}{l}21(30.4) \\
65(27.7)\end{array}$ & 0.203 & 1 & 0.653 \\
\hline \multicolumn{14}{|l|}{ Previous BCS attempt } \\
\hline $\begin{array}{l}\text { Yes } \\
\text { No }\end{array}$ & $\begin{array}{c}54(17.8) \\
250(82.2)\end{array}$ & $\begin{array}{c}25(46.3) \\
125(50.0)\end{array}$ & 0.244 & 1 & 0.622 & $\begin{array}{l}28(51.9) \\
135(54 .)\end{array}$ & 0.082 & 1 & 0.774 & $\begin{array}{l}16(29.6) \\
70(28.0)\end{array}$ & 0.058 & 1 & 0.809 \\
\hline \multicolumn{14}{|l|}{ BCS completed stage } \\
\hline $\begin{array}{l}\text { Written stage } \\
\text { Preliminary stage } \\
\text { None }\end{array}$ & $\begin{array}{c}5(1.6) \\
44(14.5) \\
255\end{array}$ & $\begin{array}{c}2(40.0) \\
21(47.7) \\
127(49.8)\end{array}$ & 0.242 & 2 & 0.886 & $\begin{array}{c}3(60.0) \\
23(52.3) \\
137(53.7)\end{array}$ & 0.115 & 2 & 0.944 & $\begin{array}{c}2(40.0) \\
12(27.3) \\
86(28.3)\end{array}$ & 0.361 & 2 & 0.835 \\
\hline \multicolumn{14}{|l|}{ Reasons for BCS preference* } \\
\hline Security of job & $145(47.7)$ & $55(37.9)$ & 14.442 & 1 & 0.001 & $72(49.7)$ & 1.751 & 1 & 0.186 & $33(22.8)$ & 4.180 & 1 & 0.041 \\
\hline High salary & $143(47.0)$ & $70(49.0)$ & 0.017 & 1 & 0.898 & $75(52.4)$ & 0.149 & 1 & 0.700 & $35(24.5)$ & 1.936 & 1 & 0.164 \\
\hline More scope of practicing power & 105 (34.5) & $56(53.3)$ & 1.022 & 1 & 0.313 & $57(54.3)$ & 0.029 & 1 & 0.865 & $27(25.7)$ & 0.524 & 1 & 0.469 \\
\hline Easier working environment & 89 (29.3) & $45(50.6)$ & 0.75 & 1 & 0.784 & $50(56.2)$ & 0.332 & 1 & 0.564 & $22(24.7)$ & 0.791 & 1 & 0.374 \\
\hline Family and social pressure & $25(8.2)$ & $20(80.0)$ & 10.243 & 1 & 0.001 & $15(60.0)$ & 0.446 & 1 & 0.504 & 8 (32.0) & 0.185 & 1 & 0.667 \\
\hline To serve the nation & $133(43.8)$ & $61(45.9)$ & 1.144 & 1 & 0.285 & $70(53.6)$ & 0.093 & 1 & 0.761 & $30(22.6)$ & 3.831 & 1 & 0.050 \\
\hline \multicolumn{14}{|l|}{ Preferred cadre of BCS } \\
\hline $\begin{array}{l}\text { Administration } \\
\text { Education } \\
\text { Police } \\
\text { Others }\end{array}$ & $\begin{array}{c}168(55.3) \\
103(33.9) \\
22(7.2) \\
11(3.6)\end{array}$ & $\begin{array}{l}85(50.6) \\
45(43.7) \\
13(59.1) \\
7(63.6)\end{array}$ & 3.158 & 3 & 0.368 & $\begin{array}{l}92(54.8) \\
58(56.3) \\
9(40.9) \\
4(36.4)\end{array}$ & 3.134 & 3 & 0.371 & $\begin{array}{l}47(28.0) \\
27(16.2) \\
7(31.8) \\
5(45.5)\end{array}$ & 1.960 & 3 & 0.581 \\
\hline \multicolumn{14}{|l|}{ Depression, anxiety, and stress } \\
\hline \multicolumn{14}{|l|}{ Depression status } \\
\hline $\begin{array}{l}\text { No } \\
\text { Yes }\end{array}$ & $\begin{array}{l}154(50.7) \\
150(49.3)\end{array}$ & - & - & - & - & $\begin{array}{l}80(51.9) \\
83(55.3)\end{array}$ & 0.350 & 1 & 0.554 & $\begin{array}{c}15(9.7) \\
71(47.3)\end{array}$ & 52.935 & 1 & 0.000 \\
\hline \multicolumn{14}{|l|}{ Anxiety status } \\
\hline $\begin{array}{l}\text { No } \\
\text { Yes }\end{array}$ & $\begin{array}{l}141(46.4) \\
163(53.6)\end{array}$ & $\begin{array}{l}67(47.5) \\
83(27.3)\end{array}$ & 0.350 & 1 & 0.554 & $\begin{array}{l}- \\
-\end{array}$ & - & $\begin{array}{l}- \\
-\end{array}$ & $\begin{array}{l}- \\
-\end{array}$ & $\begin{array}{l}37(26.2) \\
49(30.1)\end{array}$ & 0.544 & 1 & 0.461 \\
\hline \multicolumn{14}{|l|}{ Stress status } \\
\hline $\begin{array}{l}\text { No } \\
\text { Yes }\end{array}$ & $218(71.7)$ & 79 (36.2) & 52.935 & 1 & 0.000 & $114(52.3)$ & 0.544 & 1 & 0.461 & - & - & - & - \\
\hline
\end{tabular}

*Multiple response. 
TABLE 2 | Binary regression analysis of the variables by depression, anxiety, and stress.

\begin{tabular}{|c|c|c|c|c|c|c|c|c|c|}
\hline \multirow[t]{2}{*}{ Variables } & \multicolumn{3}{|c|}{ Depression } & \multicolumn{3}{|c|}{ Anxiety } & \multicolumn{3}{|c|}{ Stress } \\
\hline & OR & $95 \% \mathrm{Cl}$ & p-value & OR & $95 \% \mathrm{Cl}$ & p-value & OR & $95 \% \mathrm{Cl}$ & $\mathrm{p}$-value \\
\hline \multicolumn{10}{|l|}{ Gender } \\
\hline Female & 1.464 & (0.929-2.308) & 0.101 & 1.229 & $(0.781-1.936)$ & 0.373 & 1.386 & (0.833-2.308) & 0.209 \\
\hline Male & \multicolumn{3}{|c|}{ Reference } & \multicolumn{2}{|c|}{ Reference } & \multicolumn{4}{|c|}{ Reference } \\
\hline \multicolumn{10}{|l|}{ Permanent residence } \\
\hline Village area & 0.870 & $(0.486-1.559)$ & 0.641 & 1.544 & $(0.860-2.773)$ & 0.146 & 1.116 & $(0.580-2.147)$ & 0.742 \\
\hline City area & \multicolumn{3}{|c|}{ Reference } & \multicolumn{2}{|c|}{ Reference } & \multicolumn{4}{|c|}{ Reference } \\
\hline \multicolumn{10}{|l|}{ Relationship status } \\
\hline Married & 0.903 & $(0.468-1.743)$ & 0.868 & 0.952 & $(0.494-1.836)$ & 0.593 & 1.001 & (0.495-2.006) & 0.361 \\
\hline In a relationship & 1.125 & (0.607-2.086) & & 1.364 & $(0.728-2.553)$ & & 0.583 & $(0.275-1.239)$ & \\
\hline Single & \multicolumn{3}{|c|}{ Reference } & \multicolumn{2}{|c|}{ Reference } & \multicolumn{4}{|c|}{ Reference } \\
\hline \multicolumn{10}{|l|}{ Socioeconomic status } \\
\hline Lower class & 1.518 & (0.736-3.132) & 0.485 & 0.777 & $(0.376-1.605)$ & 0.559 & 1.163 & $(0.549-2.464)$ & 0.083 \\
\hline Middle class & 1.078 & $(0.622-1.868)$ & & 0.738 & $(0.423-1.285)$ & & 0.606 & $(0.333-1.103)$ & \\
\hline Upper class & & ference & & & ference & & & ference & \\
\hline Graduation institute & & & & & & & & & \\
\hline College & 0.930 & (0.590-1.466) & 0.754 & 1.065 & $(0.675-1.681)$ & 0.786 & 1.181 & (0.710-1.964) & 0.521 \\
\hline University & & ference & & & ference & & & eference & \\
\hline Graduation year & & & & & & & & & \\
\hline 2015 to 2012 & 1.411 & (0.730-2.730) & 0.166 & 0.955 & $(0.494-1.847)$ & 0.490 & 2.060 & $(1.018-4.171)$ & 0.244 \\
\hline 2016 & 0.596 & $(0.304-1.167)$ & & 0.601 & (0.311-1.162) & & 1.214 & (0.574-2.568) & \\
\hline 2017 & 1.139 & $(0.649-1.997)$ & & 0.874 & $(0.497-1.538)$ & & 1.397 & $(0.740-2.638)$ & \\
\hline 2018 & & ference & & & ference & & & eference & \\
\hline Current part-time job & & & & & & & & & \\
\hline Yes & 0.795 & $(0.464-1.363)$ & 0.405 & 2.385 & $(1.345-4.230)$ & 0.003 & 1.144 & (0.636-2.058) & 0.653 \\
\hline No & & ference & & & ference & & & eference & \\
\hline Previous BCS attempt & & & & & & & & & \\
\hline Yes & 0.862 & $(0.478-1.554)$ & 0.622 & 0.917 & $(0.509-1.653)$ & 0.774 & 1.083 & $(0.567-2.066)$ & 0.810 \\
\hline No & & eference & & & ference & & & eference & \\
\hline BCS completed stage & & & & & & & & & \\
\hline Written stage & 0.672 & (0.110-4.089) & 0.887 & 1.292 & $(0.212-7.863)$ & 0.944 & 1.694 & $(0.277-10.352)$ & 0.838 \\
\hline Preliminary stage & 0.920 & $(.485-1.746)$ & & 0.943 & $(0.497-1.790)$ & & 0.953 & $(0.465-1.953)$ & \\
\hline None & & eference & & & ference & & & eference & \\
\hline Reasons for BCS preference & & & & & & & & & \\
\hline Security of job & 0.412 & $(0.260-0.653)$ & $<0.001$ & 0.737 & (0.469-1.159) & 0.186 & 0.589 & $(0.354-0.981)$ & 0.042 \\
\hline High salary & 0.971 & $(0.619-1.523)$ & 0.898 & 0.915 & $(0.582-1.437)$ & 0.700 & 0.699 & $(0.422-1.159)$ & 0.165 \\
\hline More scope of practicing power & 1.277 & $(0.795-2.050)$ & 0.312 & 1.042 & $(0.648-1.674)$ & 0.865 & 0.821 & $(0.482-1.400)$ & 0.469 \\
\hline Easier working environment & 1.071 & $(0.654-1.756)$ & 0.784 & 1.157 & $(0.704-1.902)$ & 0.565 & 0.775 & $(0.441-1.361)$ & 0.375 \\
\hline Family \& social pressure & 4.585 & $(1.673-12.560)$ & $<0.001$ & 1.328 & $(0.577-3.057)$ & 0.505 & 1.213 & (0.503-2.924) & 0.668 \\
\hline To serve the nation & 0.781 & $(0.496-1.229)$ & 0.285 & 0.932 & (0.592-1.468) & 0.761 & 0.598 & (0.357-1.003) & 0.050 \\
\hline Preferred cadre of BCS & & & & & & & & & \\
\hline Administration & 0.585 & $(0.165-2.074)$ & 0.374 & 2.118 & $(0.598-7.509)$ & 0.384 & 0.466 & $(0.136-1.601)$ & 0.596 \\
\hline Education & 0.443 & $(0.122-1.608)$ & & 2.256 & (0.622-8.183) & & 0.426 & $(0.120-1.511)$ & \\
\hline Police & 0.801 & $(0.185-3.676)$ & & 1.212 & $(0.272-5.396)$ & & 0.560 & $(0.126-2.479)$ & \\
\hline Others & & ference & & & ference & & & eference & \\
\hline Depression status & & & & & & & & & \\
\hline Yes & - & - & - & 1.146 & (0.730-1.799) & 0.554 & 8.328 & $(4.473-15.507)$ & $<0.001$ \\
\hline No & - & - & - & & ference & & & ference & \\
\hline Anxiety status & & & & & & & & & \\
\hline Yes & 1.146 & (0.730-1.799) & 0.554 & - & - & - & 1.208 & (0.731-1.998) & 0.461 \\
\hline No & & ference & & - & - & - & & eference & \\
\hline Stress status & & & & & & & & & \\
\hline Yes & 8.328 & $(4.473-15.507)$ & $<0.001$ & 1.208 & $(0.731-1.998)$ & 0.461 & - & - & - \\
\hline No & & ference & & & ference & & - & - & - \\
\hline
\end{tabular}

anxiety, and stress, and unemployment in this highly educated sector of the population.

High rates of depression, anxiety, and stress were detected in this cohort. Unfortunately, since there are no other studies among job-seeking university graduates in Bangladesh, more rigorous comparisons to elucidate temporal trends or additional contributing factors are precluded. The only study conducted among medical students in Bangladesh using the same instrument also showed high prevalence rates of depression, anxiety, and stress (54.3\%, 64.8\%, and $59.0 \%$ respectively) (33). The elevated prevalence of these issues among medical students may be explained by the significant academic, psychosocial, and existential stressors for coping imposed 
by the medical college academic curriculum and learning schedules $(33,34)$. In this setting, the prevalence rates reported from other countries around the world have revealed substantial variability suggesting that both local social and cultural factors, as well as the underlying common elements promoting the emergence of depression, anxiety, and stress, may lead to marked heterogeneity in the prevalence rates of these psychiatric disturbances. Notwithstanding, higher prevalence of these psychiatric symptoms is universally more likely to occur among unemployed graduates all over the world (2-6). Previous studies have reported elevated rates of depression, anxiety, and stress among involuntarily unemployed individuals. For example, the following rates were estimated in the US: depression $[\mathrm{D}]=29 \%$, anxiety $[\mathrm{A}]=31 \%$, and stress $[\mathrm{S}]=$ $28 \%$ (35). Similarly, among unemployed adults after the economic crisis in Greece: $\mathrm{D}=32.2 \% ; \mathrm{A}=39.7 \%$, and $\mathrm{S}=33 \%$ (15); in Spain: $\mathrm{D}=51.5 \%$ and $\mathrm{A}=35.5 \%$ (36); among unemployed university graduates in Korea: $\mathrm{D}=39.5 \%$ (3); among unemployed graduates in the UK: $\mathrm{S}=69.4 \%$ (2); and finally, among unemployed people in Denmark: $S=10.4 \%$ (6). Moreover, studies conducted in neighboring countries using DASS-21 showed that the prevalence of these problems was also high among college students in Pakistan, $\mathrm{D}=35.9 \%, \mathrm{~A}=64 \%$, and $\mathrm{S}=38.5 \%$ (37); among medical students in India, $\mathrm{D}=32.0 \%, \mathrm{~A}=40.1 \%$, and $\mathrm{S}=43.8 \%$ (38); and among medical students in Nepal: $\mathrm{D}=29.9 \%, \mathrm{~A}=41.1 \%$, and $\mathrm{S}=27 \%$ (39). Additionally, across the world, the rate varies, with a wide range among university students [such as $\mathrm{D}=37.2 \%, \mathrm{~A}=63 \%$, and $\mathrm{S}=23.7 \%$ in Malaysia (40); $\mathrm{D}=27.1 \%, \mathrm{~A}=47.1 \%$, and $\mathrm{S}=27 \%$ in Turkey (41); and D $=23 \%, A=25 \%$, and $S=26 \%$ in the United States (42)]. (Please see Online Supplemental Table.)

In general, women are more likely to experience job-seeking stress and depression compared to men (7). Moreover, when women were also single, the latter seemed to operate as a mediator of depression (15), while conversely, being married appeared to dampen the likelihood of depression among unemployed women while imposing the reverse effect in men (7). Of note, we found no significant associations between mental health symptoms and gender, even when incorporating marital relationship status in the analyses.

Lower-socioeconomic-group participants are also more likely to experience mental health symptoms when compared to higher socioeconomic groups $(32,43)$. Thus, economic distress along with family and social pressure for job attainment fosters the emergence of mental health problems $(2,4-6)$. Younger job seekers who are willing to accept irregular employment (3) or have chosen to take a stopgap job that does not match their qualifications and skill level experienced greater psychiatric problems (44). In a study among Bangladeshi garment workers, part-time employment was found to be associated with depressive disorders (32). In the present study, participants who were engaged in a part-time job were at higher risk of reporting symptoms of anxiety. This may possibly due to the pressure of family poverty fostering economic uncertainty and associated anxiety.

Moreover, achievement motivation may play also play a role in anxiety, since unemployed individuals may be retain increased hope of securing the desired job, while the underemployed may have partially given up hope and accepted failure as their reality (2). However, repeated previous attempts in the BCS examination and failure at the higher stages (mentioned hierarchically as viva, written, and preliminary stage) did not seem to exert any role in the occurrence of mental disturbances compared to those who had never taken the exam. Thus, sustained joblessness and failure of getting a job would be anticipated to foster mental impairments and decrease well-being and quality of life, ultimately leading to depression, anxiety, and stress $(3,4)$.

There is consistent evidence that losing job security, which can play a role in UUE at any time, will impose significant adverse effects on psychological co-morbidity and disturbances $(2,4,45)$. Therefore, study participants who were insecure about the BCS job were more likely to suffer from depression and stress. Moreover, participants reporting family and social pressures to become a BCS cadre were more likely to suffer from depression, as perceived social pressure and stress accelerate the onset of depressive disorders (2). Conversely, social support acts as an important factor to decrease job-searching stress as well as to prevent depression (3). Interestingly, choosing BCS jobs with the aim of serving the nation reflected as a negative predictor of stress. More altruistic goals and potentially less socioeconomic pressure may underlie this observation.

\section{LIMITATIONS OF THE STUDY}

There are several limitations to this study that deserve mention. Firstly, our study was cross-sectional in nature, and therefore, a stable and potentially causal relationship between depression, anxiety, stress, and their socio-demographic correlates cannot be ascertained. The findings are also limited by the self-reported questionnaire responses, which might be influenced in the context of method bias, memory recall biases, and social desirability biases. Such biases can be potentially removed in future studies through implementation of different methodologies. The relatively small sample size should also be viewed as a potential limitation. Furthermore, the present study was conducted exclusively in the Rajshahi region of the country, such that generalizability may not necessarily be possible.

\section{IMPLICATIONS OF THE STUDY}

This is the first study in Bangladesh focusing on common mental health problems and university graduate unemployment. Similar to other studies around the world, a large portion of young people are struggling to cope with a new stage of their professional life after completion of their studies and are at high risk of suffering from various psychiatric distresses, like depression, anxiety, and stress (3, $5,7)$, whereas securing a suitable and desirable job has significant benefits for mental well-being (2). We should emphasize that the presence of mental disturbances can affect both productivity and prosperity in life and even increase the risk of suicide and suicidal behaviors (12-14). Therefore, special attention to the mental health of unemployed graduates appears mandatory and is predicated on thorough evaluation of predictive risk factors, which will have to be further explored by more expansive and longitudinal interventional studies. Technical, polytechnic, and vocational education venues can also play a crucial role in reducing unemployment by training 
graduates and secure them employment aligned with their training through better matching of education and job markets. Furthermore, in the setting of high demands for technically and vocationally trained graduates, job opportunities can be leveraged not only within the country but also abroad, a situation that was adopted in China and recently reported (1).

\section{CONCLUSIONS}

The present study reports on the high prevalence and associated risk factors of depression, anxiety, and stress among BCS job seekers. The presence of job insecurity and family and social pressures to secure a BCS job are strong predictors for depression, while being already involved in a part-time job emerged as a risk factor for anxiety. In contrast, intent of obtaining a BCS position to serve the nation predicted reduced stress. This study indicates that preventive workforce initiatives aimed at better alignment between educational pipelines and job markets are needed. Furthermore, early mental support and resilience training programs during higher education are needed to potentially mitigate the elevated risk of mental issues among university graduates in Bangladesh by further exploring with a national large-scale sample concerning our yielded risk issues.

\section{DATA AVAILABILITY}

The datasets generated for this study are available on request to the corresponding author.

\section{ETHICS STATEMENT}

This study was carried out in accordance with the recommendations of "name of guidelines, name of committee"

\section{REFERENCES}

1. Wadud M. Sufferings and solutions of unemployment. Prothom Alo (2018).

2. Cassidy T, Wright L. Graduate employment status and health: a longitudinal analysis of the transition from student. Soc Psychol Educ (2008) 11:181-91. doi: $10.1007 /$ s11218-007-9043-x

3. Lim AY, Lee S-H, Jeon Y, Yoo R, Jung H-Y. Job-seeking stress, mental health problems, and the role of perceived social support in university graduates in Korea. J Korean Med Sci (2018) 33:149. doi: 10.3346/jkms. 2018.33.e149

4. Meltzer H, Bebbington P, Brugha T, Jenkins R, McManus S, Stansfeld S. Job insecurity, socio-economic circumstances and depression. Psychol Med (2010) 40:1401-7. doi: 10.1017/S0033291709991802

5. Reneflot A, Evensen M. Unemployment and psychological distress among young adults in the Nordic countries: a review of the literature. Int J Soc Welf (2014) 23:3-15. doi: 10.1111/ijsw.12000

6. Mæhlisen MH, Pasgaard AA, Mortensen RN, Vardinghus-Nielsen H, TorpPedersen C, Bøggild H. Perceived stress as a risk factor of unemployment: a register-based cohort study. BMC Public Health (2018) 18:728. doi: 10.1186/ s12889-018-5618-z

7. Artazcoz L, Benach J, Borrell C, Cortès I. Unemployment and mental health: understanding the interactions among gender, family roles, and social class. Am J Public Health (2004) 94:82-8. doi: 10.2105/AJPH.94.1.82 with written informed consent from all subjects. All subjects gave written informed consent in accordance with the Declaration of Helsinki. The protocol was approved by the Institutional Review Board of the Institute of Allergy and Clinical Immunology of Bangladesh (IACIB), Savar, Dhaka, Bangladesh (Ref. No: IRBIACIB/CEC/07201801).

\section{AUTHOR CONTRIBUTIONS}

MAR, MM, KH, and MH conceptualized the initial phases of the study, collected and analyzed data, and drafted initial versions of the manuscript. DG contributed to analyses and performed editing of the manuscript for content and style. All authors have approved the final version of the manuscript.

\section{FUNDING}

DG is supported by National Institutes of Health grants 1R01HL130984 and R56 HL140548.

\section{ACKNOWLEDGMENTS}

The authors would like to thank the World Linkup team (of Rajshahi University, Rajshahi, Bangladesh) for their support during data collection.

\section{SUPPLEMENTARY MATERIAL}

The Supplementary Material for this article can be found online at: https://www.frontiersin.org/articles/10.3389/fpsyt.2019.00578/ full\#supplementary-material

8. Arafat SMY. Psychological autopsy study in Bangladesh: AN unmet need to formulate preventive strategy of suicide. Asian J Psychiatr (2019) 43:85-86. doi: 10.1016/j.ajp.2019.05.019

9. Harris EC, Barraclough B. Suicide as an outcome for mental disorders. A meta-analysis. Br J psychiatry (1997) 170:205-28. doi: 10.1192/ bjp.170.3.205

10. Fleischmann A, Bertolote JM, Belfer M, Beautrais A. Completed suicide and psychiatric diagnoses in young people: a critical examination of the evidence. Am J Orthopsychiatry (2005) 75:676-83. doi: 10.1037/0002-9432.75.4.676

11. Arafat SMY, Mamun MAA. Repeated suicides in the University of Dhaka (November 2018): strategies to identify risky individuals. Asian J Psychiatr (2019) 39:84-5. doi: 10.1016/j.ajp.2018.12.014

12. Pompili M, Innamorati M, Di Vittorio C, Baratta S, Masotti V, Badaracco A, et al. Unemployment as a risk factor for completed suicide: a psychological autopsy study. Arch Suicide Res (2014) 18:181-92. doi: 10.1080/13811118. 2013.803449

13. Nordt C, Warnke I, Seifritz E, Kawohl W. Modelling suicide and unemployment: a longitudinal analysis covering 63 countries, 2000-11. Lancet Psychiatry (2015) 2:239-45. doi: 10.1016/S2215-0366(14)00118-7

14. Milner A, Page A, LaMontagne AD. Long-term unemployment and suicide: a systematic review and meta-analysis. PLoS One (2013) 8:e51333. doi: 10.1371/journal.pone.0051333 
15. Kokaliari E. Quality of life, anxiety, depression, and stress among adults in Greece following the global financial crisis. Int Soc Work (2018) 61:410-24. doi: $10.1177 / 0020872816651701$

16. New Age. Unemployment among univ graduates rises. New Age Bangladesh (2018).

17. The Daily Star. (2018). 38th BCS preliminary results published. Available at: https://www.thedailystar.net/country/38th-bcs-preliminary-examinationresult-2018-published-bangladesh-public-service-commissionbpsc-1541398 [Accessed January 10, 2019]

18. World Health Organization. Depression and other common mental disorders: global health estimates. (2017). https://www.who.int/mental_health/ management/depression/prevalence_global_health_estimates/en/

19. Hossain MD, Ahmed HU, Chowdhury WA, Niessen LW, Alam DS. Mental disorders in Bangladesh: a systematic review. BMC Psychiatry (2014) 14:8. doi: 10.1186/s12888-014-0216-9

20. bbdnews24.com. Tanvir Rahman killed himself after failing to get government job: family. (2018) Available at: https://bdnews24.com/bangladesh/2018/04/01/ tanvir-rahman-killed-himself-after-failing-to-get-government-job-family [Accessed October 10, 2019].

21. Bangladesh Public Service Commission. বসিএিস পরীকয়া. (2018) Available at: http://www.bpsc.gov.bd/site/page/4bc95017-18d6-412b-8c4f-76d3e1599d8e/ বসিত্রিস-পরীকযা [Accessed January 10, 2019]

22. Lovibond PF, Lovibond SH. Manual for the Depression Anxiety Stress Scales. 2nd. Sydney: Psychology Foundation (1995). doi: 10.1037/t01004-000

23. Alim SMAHM, Kibria SME, lslam MJ, Uddin MZ, Nessa M, Wahab MA, et al. Translation of DASS 21 into Bangla and validation among medical students. Bangladesh J Psychiatry (2016) 28:67-70. doi: 10.3329/bjpsy.v28i2.32740

24. Mamun MAA, Griffiths MD. The association between Facebook addiction and depression: a pilot survey study among Bangladeshi students. Psychiatry Res (2019) 271:628-33. doi: 10.1016/j.psychres.2018.12.039

25. Roy T, Lloyd CE, Parvin M, Mohiuddin KGB, Rahman M. Prevalence of co-morbid depression in out-patients with type 2 diabetes mellitus in Bangladesh. BMC Psychiatry (2012) 12:123. doi: 10.1186/1471-244X-12-123

26. Islam MA, Rahman A, Aleem MA, Islam SMS. Prevalence and associated factors of depression among post-stroke patients in Bangladesh. Int J Ment Health Addict (2016) 14:154-66. doi: 10.1007/s11469-015-9582-x

27. Mamun MA, Huq N, Papia ZF, Tasfina S, Gozal D. Prevalence of depression among Bangladeshi village women subsequent to a natural disaster: a pilot study. Psychiatry Res (2019) 276:124-8. doi: 10.1016/j. psychres.2019.05.007

28. Mamun MA, Rafi MA, Al Mamun AHMS, Hasan MZ, Akter K, Hsan K, et al. Prevalence and psychiatric risk factors of excessive internet use among northern Bangladeshi job-seeking graduate students: a pilot study. Int J Ment Health Addict (2019) doi: 10.1007/s11469-019-00066-5

29. Asghar S, Hussain A, Ali SMK, Khan AKA, Magnusson A. Prevalence of depression and diabetes: a population based study from rural Bangladesh. Diabet Med (2007) 24:872-7. doi: 10.1111/j.1464-5491.2007.02136.x

30. Arafat SMY, Mamun MAA, Uddin MS. Depression literacy among first-year university students: a cross-sectional study in Bangladesh. Glob Psychiatry (2019) 2:31-6. doi: 10.2478/gp-2019-0002

31. Mamun MA, Hossain MS, Siddique AB, Sikder MT, Kuss DJ, Griffiths MD. Problematic internet use in Bangladeshi students: the role of sociodemographic factors, depression, anxiety, and stress. Asian J Psychiatr (2019) 44:48-54. doi: 10.1016/j.ajp.2019.07.005

32. Fitch TJ, Moran J, Villanueva G, Sagiraju HKR, Quadir MM, Alamgir H. Prevalence and risk factors of depression among garment workers in Bangladesh. Int J Soc Psychiatry (2017) 63:244-54. doi: 10.1177/00207640 17695576
33. Alim SMAHM, Rabbani MG, Karim E, Mullick MSI, Al Mamun A, Khan MZR. Assessment of depression, anxiety and stress among first year MBBS students of a public medical college, Bangladesh. Bangladesh J Psychiatry (2017) 29:23-9. doi: 10.3329/bjpsy.v29i1.32748

34. Kulsoom B, Afsar NA. Stress, anxiety, and depression among medical students in a multiethnic setting. Neuropsychiatr Dis Treat (2015) 11:171322. doi: 10.2147/NDT.S83577

35. Howe GW, Hornberger AP, Weihs K, Moreno F, Neiderhiser JM. Higherorder structure in the trajectories of depression and anxiety following sudden involuntary unemployment. J Abnorm Psychol (2012) 121:325-38. doi: $10.1037 / \mathrm{a} 0026243$

36. Navarro-Abal Y, Climent-Rodríguez J, López-López M, Gómez-Salgado J. Psychological coping with job loss. Empirical study to contribute to the development of unemployed people. Int J Environ Res Public Health (2018) 15:1787. doi: 10.3390/ijerph15081787

37. Nadeem M, Ali A, Buzdar MA. The association between Muslim religiosity and young adult college students' depression, anxiety, and stress. J Relig Health (2017) 56:1170-9. doi: 10.1007/s10943-016-0338-0

38. Taneja N, Sachdeva S, Dwivedi N. Assessment of depression, anxiety, and stress among medical students enrolled in a medical college of New Delhi, India. Indian J Soc Psychiatry (2018) 34:157-62.

39. Kunwar D, Risal A, Koirala S. Study of depression, anxiety and stress among the medical students in two medical colleges of Nepal. Kathmandu Univ Med J (2016) 53:22-6.

40. Shamsuddin K, Fadzil F, Ismail WSW, Shah SA, Omar K, Muhammad NA, et al. Correlates of depression, anxiety and stress among Malaysian university students. Asian J Psychiatr (2013) 6:318-23. doi: 10.1016/j. ajp.2013.01.014

41. Bayram N, Bilgel N. The prevalence and socio-demographic correlations of depression, anxiety and stress among a group of university students. Soc Psychiatry Psychiatr Epidemiol (2008) 43:667-72. doi: 10.1007/s00127008-0345-x

42. Beiter R, Nash R, McCrady M, Rhoades D, Linscomb M, Clarahan M, et al. The prevalence and correlates of depression, anxiety, and stress in a sample of college students. J Affect Disord (2015) 173:90-6. doi: 10.1016/j. jad.2014.10.054

43. Lorant V, Deliège D, Eaton W, Robert A, Philippot P, Ansseau M. Socioeconomic inequalities in depression: a meta-analysis. Am J Epidemiol (2003) 157:98-112. doi: 10.1093/aje/kwf182

44. Cassidy T. The psychological health of employed and unemployed recent graduates as a function cognitive appraisal and of coping. Couns Psychol Q (1994) 7:385-97. doi: 10.1080/09515079408254161

45. Ferrie JE, Shipley MJ, Stansfeld SA, Marmot MG. Effects of chronic job insecurity and change in job security on self reported health, minor psychiatric morbidity, physiological measures, and health related behaviours in British civil servants: THE Whitehall II study. J Epidemiol Community Heal (2002) 56:450-4. doi: 10.1136/jech.56.6.450

Conflict of Interest Statement: The authors declare that the research was conducted in the absence of any commercial or financial relationships that could be construed as a potential conflict of interest.

Copyright (๑) 2019 Rafi, Mamun, Hsan, Hossain and Gozal. This is an open-access article distributed under the terms of the Creative Commons Attribution License (CC BY). The use, distribution or reproduction in other forums is permitted, provided the original author(s) and the copyright owner(s) are credited and that the original publication in this journal is cited, in accordance with accepted academic practice. No use, distribution or reproduction is permitted which does not comply with these terms. 